\title{
Model Study of the Rupture Phenomena of a Metal Droplet Growing on a Slag-Metal Interface
}

\author{
Tsuyoshi KAGAWA and Manabu IGUCHI ${ }^{11}$ \\ Graduate Student, Graduate School of Engineering, Hokkaido University, North 13, West 8, Kita-ku, Sapporo 060-8628 Japan. \\ 1) Division of Materials Science and Engineering, Graduate School of Engineering, Hokkaido University, North 13 , West 8 , \\ Kita-ku, Sapporo 060-8628 Japan.
}

(Received on January 27, 2005; accepted on May 25, 2005)

\begin{abstract}
Cold model experiments were carried out to understand the behavior of a molten steel droplet growing on a slag and molten steel interface due to coalescence with other molten steel droplets. Water, silicone oil, and n-pentane were used as the working fluids. A water droplet was generated from a single-hole nozzle placed just above a silicone oil and water interface or an n-pentane and water interface. The droplet grew in contact with the interface and then ruptured. An empirical equation was proposed for the volume of the droplet. The flow fields in and around the droplet were visualized with a CCD camera and the velocity vectors were determined with particle image velocimetry (PIV). The flow pattern near the interface was correlated in terms of the Weber number similitude.
\end{abstract}

KEY WORDS: refining; droplet; slag; wettability; nozzle.

\section{Introduction}

In the refining processes agitated by bottom and side gas injection many molten steel droplets are carried by rising bubbles up into the molten slag layer. According to a previous study, ${ }^{1)}$ the droplets are known to be caused by breakup of a filament-like molten steel formed behind a bubble penetrating into the upper slag layer. A preliminary water model experiment reveals that when the population of the droplets is high, they coalesce successively with one another at the slag-metal interface. Furthermore, when the volume of a growing droplet exceeds a certain critical value, it ruptures at the interface and then returns to the lower molten steel layer. The behavior of the droplets in the molten slag layer and that at the interface between the molten slag and molten steel layers are closely associated with the efficiency of the processes.

Many papers can be seen on the shape of a droplet staying on a liquid-liquid interface, ${ }^{2-5}$ coalescence of two droplets, ${ }^{6,7)}$ and the dynamic behavior of a droplet passing across a liquid-liquid interface at a terminal velocity. ${ }^{8-12)}$ As far as the authors are aware, information on a rupture phenomenon of a droplet growing on a liquid-liquid interface is not available.

In this study the rupture of a molten steel droplet growing on a liquid-liquid interface due to coalescence with other droplets was investigated using a water model. However, it is difficult to model the successive coalescence of many droplets. The coalescence process therefore was further simplified. Namely, a droplet growing at the interface due to successive coalescence was modeled by a droplet growing at the exit of a single-hole nozzle placed just above the interface. Silicone oil or n-pentane was placed on a water bath. A water droplet was generated with a single-hole nozzle placed just above the interface between the two liquid layers. When the nozzle was wetted by water, the water droplet spread horizontally on the end of the nozzle and reached the outer edge of the nozzle. ${ }^{13)}$ On the other hand, the water droplet never spread toward the outer edge when the nozzle was poorly wetted by water. A single-hole nozzle of poor wettability therefore was used in this study because a droplet generated by this nozzle was closer to a droplet of spherical in shape than that generated by a wetted nozzle. The rupture of a water droplet growing on an interface between two liquid layers was observed with a highspeed video camera. The flows in and around the droplet were measured with particle image velocimetry and their velocity vectors were obtained.

\section{Experiment}

According to the previous study, ${ }^{13)}$ the flow pattern in a water droplet generated using a poorly wetted single-hole nozzle can be correlated as a function of the Bond number, Bo, and a modified Weber number, $\mathrm{We}^{\prime}$, as shown in Fig. 1.

$$
\begin{gathered}
\mathrm{Bo}=\Delta \rho g d_{\mathrm{ni}}^{2} / \gamma \quad \ldots \\
\mathrm{We}^{\prime}=\Delta \rho Q_{\mathrm{w}} v_{\mathrm{n}} /\left(\gamma d_{\mathrm{ni}}\right) \\
\Delta \rho=\rho_{\mathrm{w}}-\rho_{\mathrm{s}}
\end{gathered}
$$

where $\Delta \rho$ is the density difference, $g$ is the acceleration due 


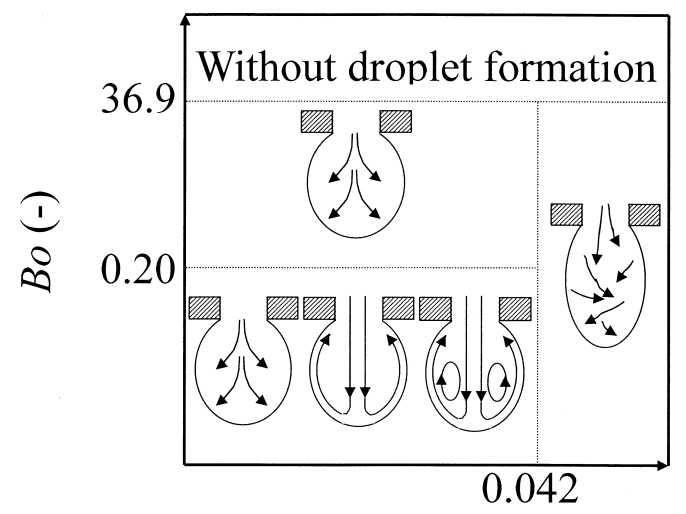

\section{$W e^{\prime}(-)$}

Fig. 1. Flow pattern in a droplet.

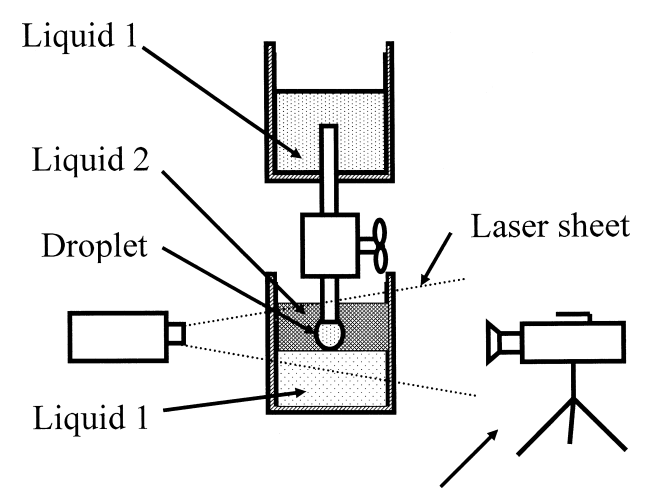

High-speed

video camera

Fig. 2. Experimental apparatus.

to gravity, $d_{\mathrm{ni}}$ is the inner diameter of the nozzle, $\gamma$ is the interfacial tension, $\rho_{\mathrm{w}}$ is the density of water, $Q_{\mathrm{w}}$ is the flow rate of water issuing out of the nozzle, $v_{\mathrm{n}}$ is the mean velocity of water, and $\rho_{\mathrm{s}}$ is the density of silicone oil or n-pentane. A water droplet was not generated for $\mathrm{Bo}>36.9$. A recirculating flow was established in the droplet for $\mathrm{Bo}<0.2$. The flow in the droplet became unstable for $\mathrm{We}^{\prime}>0.042$. In this study experiments were carried out for $0.03<$ Bo $<0.4$ and $1 \times 10^{-4}<\mathrm{We}^{\prime}<0.10$.

A schematic diagram of the experimental apparatus is shown in Fig. 2. Water was supplied from a reservoir and then introduced into a single-hole nozzle placed in a silicone oil layer or an n-pentane layer. The flow rate of water was controlled with a valve and a rotameter. The nozzle was placed just above the interface so that a water droplet generated at the nozzle exit grew on the interface and then ruptured. The distance from the nozzle to the interface was denoted by $H_{\mathrm{n}}$. As already mentioned in the introduction, this process is a simplified model for the growth of a molten steel droplet on the slag-metal interface due to coalescence with other droplets. Namely, the coalescence position of the droplets was assumed to be located at the nozzle exit.

A nozzle made of glass was originally wetted by water. The end of the nozzle was polished with a sheet of fine
Table 1. Physical properties of liquids.

\begin{tabular}{|l|c|c|}
\multicolumn{3}{|c}{ Liquid 1: water } \\
\hline & $\begin{array}{c}\Delta \rho: \text { density } \\
\text { difference } \\
\left(\mathrm{kg} / \mathrm{m}^{3}\right)\end{array}$ & $\begin{array}{c}\gamma: \text { interfacial } \\
\text { tension } \\
(\mathrm{mN} / \mathrm{m})\end{array}$ \\
\hline silicone oil & 179 & 56.1 \\
\hline n-pentane & 367 & 57.3 \\
\hline
\end{tabular}

sand paper and then coated with repellent to change its wettabilty. The surface thus coated was poorly wetted by water. The contact angle was $142^{\circ}$. The inner diameter of the nozzle, $d_{\text {ni }}$, was $1.0 \mathrm{~mm}$ and $2.5 \mathrm{~mm}$. The outer diameter of the nozzle, $d_{\text {no }}$, was kept at $8.0 \mathrm{~mm}$.

The rupture of a water droplet growing on the interface was observed with a high-speed video camera at 500 frames/s. The velocity vectors of the flows in and around the droplet were measured with particle image velocimetry. The density difference and interfacial tension were $\Delta \rho=$ $179 \mathrm{~kg} / \mathrm{m}^{3}$ and $\gamma=56.1 \mathrm{mN} / \mathrm{m}$, respectively for the water and silicone oil system and $\Delta \rho=367 \mathrm{~kg} / \mathrm{m}^{3}$ and $\gamma=57.3$ $\mathrm{mN} / \mathrm{m}$, respectively for the water and $\mathrm{n}$-pentane system, as seen in Table 1. The kinematic viscosities of water, silicone oil, and n-pentane were $0.891 \mathrm{~mm}^{2} / \mathrm{s}, 1.0 \mathrm{~mm}^{2} / \mathrm{s}$ and 0.36 $\mathrm{mm}^{2} / \mathrm{s}$, respectively. In this study only the effects of density difference and kinematic viscosity of the upper liquid, i.e., liquid 2 on the rupture of the droplet were investigated because the interfacial tensions were approximately the same for the two systems.

A water droplet was also generated at the exit of a nozzle placed far above the interface. The shape and size of the droplet were observed with the high-speed video camera. The results were compared with those of a droplet growing on the interface. Tracer particles of a mean diameter of $11 \mu \mathrm{m}$ were mixed in the water droplet to visualize the flow field in and around the water droplet. Particular attention was paid to the water flow penetrating into the lower water layer after the rupture of the water droplet.

\section{Experimental Results and Discussion}

\subsection{Shape and Size of Water Droplet}

Figure 3 shows photographs of a water droplet generated far above the interface and one growing on the interface. In the latter case the motion of the droplet in the downward direction was suppressed by an interfacial tension force acting on a silicone oil film formed between the droplet and the lower water layer. Accordingly, the droplet spread in the horizontal direction until it reached a certain position and then ruptured to result in contact with the lower water layer.

Figures $\mathbf{4}$ and $\mathbf{5}$ show the relationship between the maximum width difference for rupture, $W_{\max }^{\prime}-W_{\max }$, and the water flow rate, $Q_{\mathrm{w}}$, for the water and silicone oil system and the water and n-pentane system, respectively. In these figures $W_{\max }^{\prime}$ is the maximum width of a water droplet growing on the interface and $W_{\max }$ is the maximum width of a water droplet generated far above the interface. The distance between the nozzle and interface, $H_{\mathrm{n}}$, was varied from 5 to $8 \mathrm{~mm}$, as shown in Fig. 4. When $H_{\mathrm{n}}$ was smaller than $5 \mathrm{~mm}$, the interface became unstable and wave motions appeared on the interface. It was difficult under this condition to judge the width of the droplet precisely. On the other 


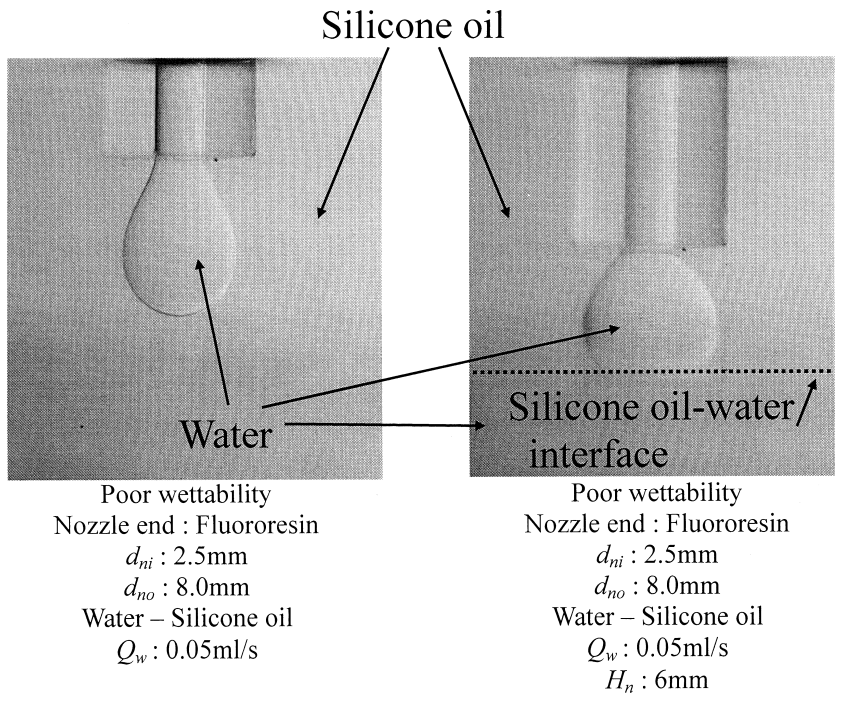

Fig. 3. Droplet generated from a nozzle placed far above a liquid-liquid interface and that growing on the interface.
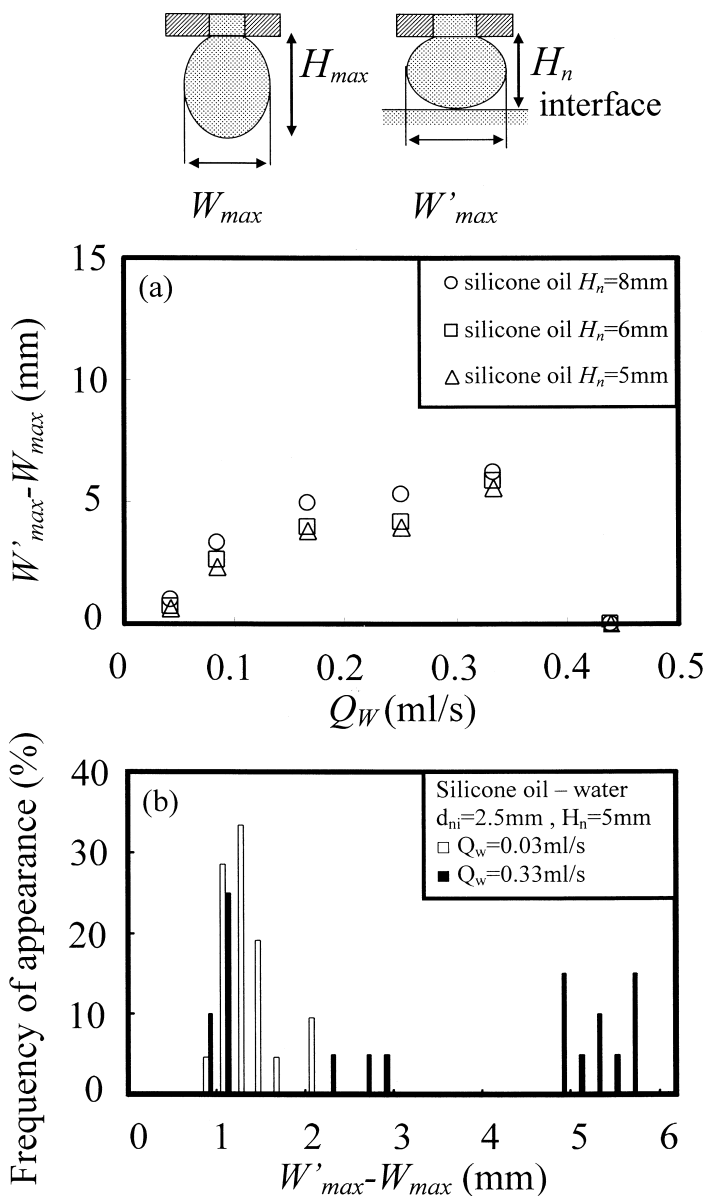

Fig. 4. Maximum width difference, $W_{\max }^{\prime}-W_{\max }$, for silicone oil and water system. (a) Effect of water flow rate, $Q_{w}$, (b) appearance frequency.

hand, when $H_{\mathrm{n}}$ was greater than $8 \mathrm{~mm}$, a droplet departed from the nozzle before attaching to the surface. This is the reason why $H_{\mathrm{n}}$ was varied from 5 to $8 \mathrm{~mm}$. The maximum width difference, $W_{\max }^{\prime}-W_{\max }$, increased with an increase in $Q_{\mathrm{w}}$, exhibited a nearly constant value, and then abruptly became zero, as shown in Fig. 4(a). The constant value increased as $H_{\mathrm{n}}$ increased.

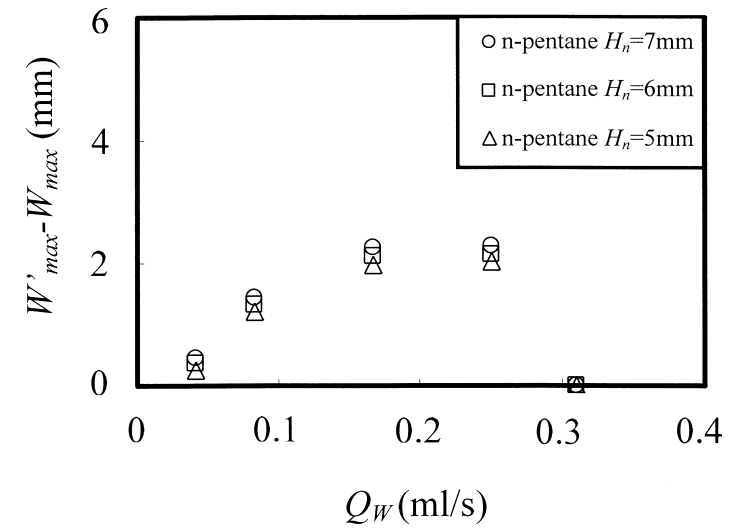

Fig. 5. Relationship between $W_{\max }^{\prime}-W_{\max }$ and water flow rate, $Q_{\mathrm{w}}$, for n-pentane and water system.

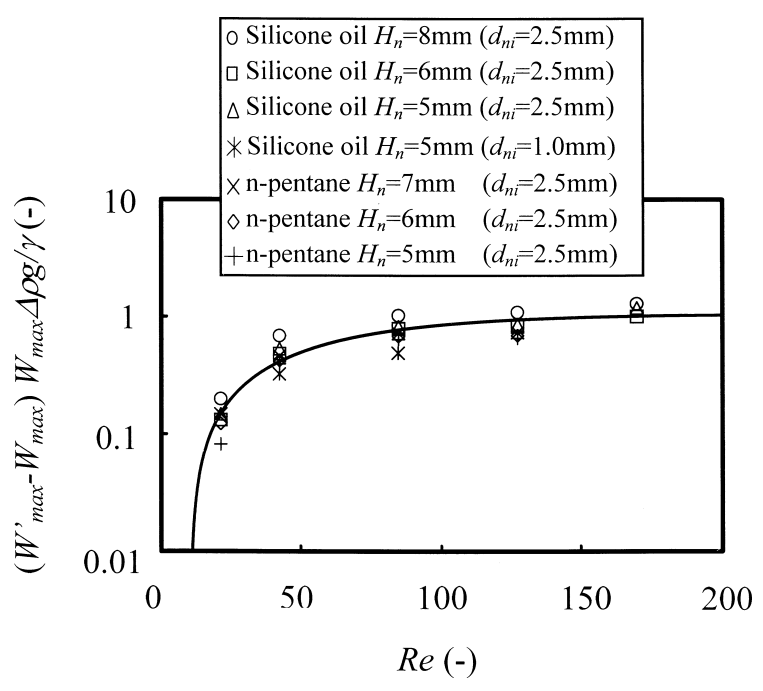

Fig. 6. Correlation of $\left(\mathrm{W}_{\max }^{\prime}-W_{\max }\right)$.

It should be noted that the appearance frequency of the maximum width difference follows a two-peak distribution as $Q_{\mathrm{w}}$ approaches a critical value of $0.33 \mathrm{ml} / \mathrm{s}$, while it follows a single-peak distribution when $Q_{\mathrm{w}}$ is low, as shown in Fig. 4(b). The maximum width difference shown in Fig. 4(a) was determined by averaging the measured values scattered around ( $W_{\max }^{\prime}-W_{\max }$ ) of $5 \mathrm{~mm}$ in Fig. 4(b).

When a water droplet approached an interface between two liquid layers, a film of the upper liquid was formed between the droplet and the lower layer. The film became thin with time because the upper liquid in the film was continuously drained. The water droplet ruptured on the interface without growing in the horizontal direction for a very low water flow rate. Under this condition the water flow rate was comparable to or smaller than the drainage rate of the upper liquid from the film. The maximum width difference of the water droplet increased with an increase in the water flow rate, as the water flow rate overcame the drainage rate. When the water flow rate exceeded a certain critical value, a water droplet ruptured as soon as it arrived at the interface, as already shown in Figs. 4 and 5. This critical value will be discussed more in detail in a later section.

Considering these circumstances, the maximum width difference, $W_{\max }^{\prime}-W_{\max }$, was non-dimensionalized and plotted against the following nozzle Reynolds number in Fig. 6. 


$$
\operatorname{Re}=d_{\mathrm{ni}} v_{\mathrm{n}} / v_{\mathrm{w}}
$$

where $v_{\mathrm{w}}$ is the kinematic viscosity of water. The measured values of the maximum width difference can be satisfactorily correlated by this arrangement method. The modified Weber number was not adequate for correlating the difference.

The following empirical equation was derived.

$$
\left(W_{\max }^{\prime}-W_{\max }\right) W_{\max } \Delta \rho g / \gamma=1.2 \tanh (0.012 \mathrm{Re})-0.15
$$

$170 \geq \operatorname{Re} \geq 21.2, \quad 1 \geq H_{\mathrm{n}} / H_{\max } \geq 0.4, \quad 0.4 \geq \mathrm{Bo} \geq 0.03$,

$0.1>\mathrm{We}^{\prime}>1 \times 10^{-4}, \quad 1.00 \mathrm{~mm}^{2} / \mathrm{s} \geq v_{\mathrm{s}} \geq 0.36 \mathrm{~mm}^{2} / \mathrm{s}$.

The kinematic viscosity of the upper liquid (liquid 2), $v_{\mathrm{s}}$, is much greater than $1 \mathrm{~mm}^{2} / \mathrm{s}$ in the real process, and accordingly, the drainage rate of the upper liquid from the film between a droplet and the lower liquid may be different from that encountered in this study. Investigation on the effect of $v_{\mathrm{s}}$ on the behavior of a droplet must be left for a future study.

Information on $W_{\max }$ and $H_{\max }$ is necessary for determining $W_{\max }^{\prime}$ from Eq. (6). By referring to the previous numerical data, ${ }^{12)}$ equations for $W_{\max }$ and $H_{\max }$ were newly derived as follows:

$$
\begin{aligned}
& W_{\max }=1.46 \mathrm{Bo}^{-0.366} d_{\mathrm{ni}} \\
& H_{\max }=2.19 \mathrm{Bo}^{-0.327} d_{\mathrm{ni}}
\end{aligned}
$$

Figure 7 shows a good comparison of the measured and numerical values for $W_{\max }$ and $H_{\max }$.

The maximum volume of a droplet being able to stay on the interface can be calculated in the following manner. The shape of the droplet generated in this experiment can be regarded as being ellipsoidal in shape. The maximum volume therefore is expressed by

$$
V_{\text {pmax }}=\pi W_{\max }^{\prime 2} H_{\mathrm{n}} / 6
$$

where $W_{\max }^{\prime}$ can be estimated from Eq. (6) and $H_{\mathrm{n}}$ is given a priori. An equivalent diameter of the droplet is given by

$$
d_{\text {pmax }}=\left(6 V_{\text {pmax }} / \pi\right)^{1 / 3}
$$

The maximum diameter of a droplet growing at a singlehole nozzle placed far above the interface, $d_{\text {pmax }}$, is expressed by ${ }^{12)}$

$$
d_{\mathrm{pmax}}=1.62 d_{\mathrm{ni}} \mathrm{Bo}^{-0.35}
$$

We assumed that $H_{\mathrm{n}}=H_{\max }$ and $\mathrm{Re}=100$ (see Fig. 6) and calculated $d_{\text {pmax }}$ for several $d_{\text {ni }}$ values. This Reynolds number value of 100 was chosen because $\left(W_{\max }^{\prime}-W_{\max }\right)$ was hardly dependent on the water flow rate, $Q_{\mathrm{w}}$, for $\operatorname{Re}>100$. The maximum diameters calculated from Eqs. (10) and (11) for the water and silicone oil system are plotted against the inner diameter of the nozzle, $d_{\text {ni }}$, in Fig. 8(a). The inner diameter, $d_{\text {ni }}$, is regarded as a dummy variable. It should be noted here that each droplet approaches the interface slowly increasing its volume continuously. The maximum diameter of a droplet growing on the interface was approximately 1.5 times as large as that generated from a nozzle placed far above the interface. This result means that any droplet growing on a liquid-liquid interface ruptures and then pen-

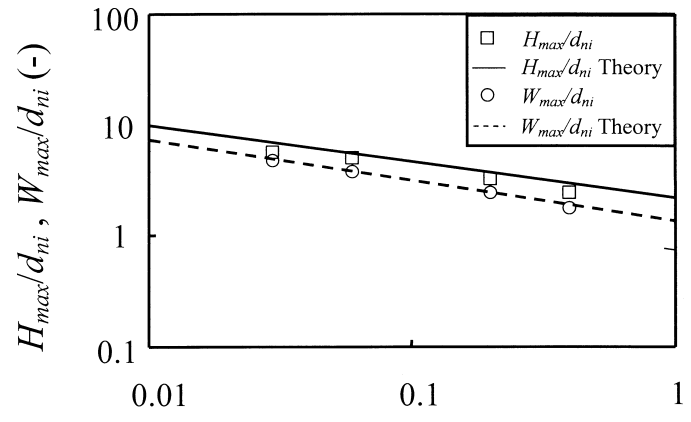

Bo (-)

Fig. 7. Maximum height and width of a droplet generated from a nozzle placed far above liquid-liquid interface.
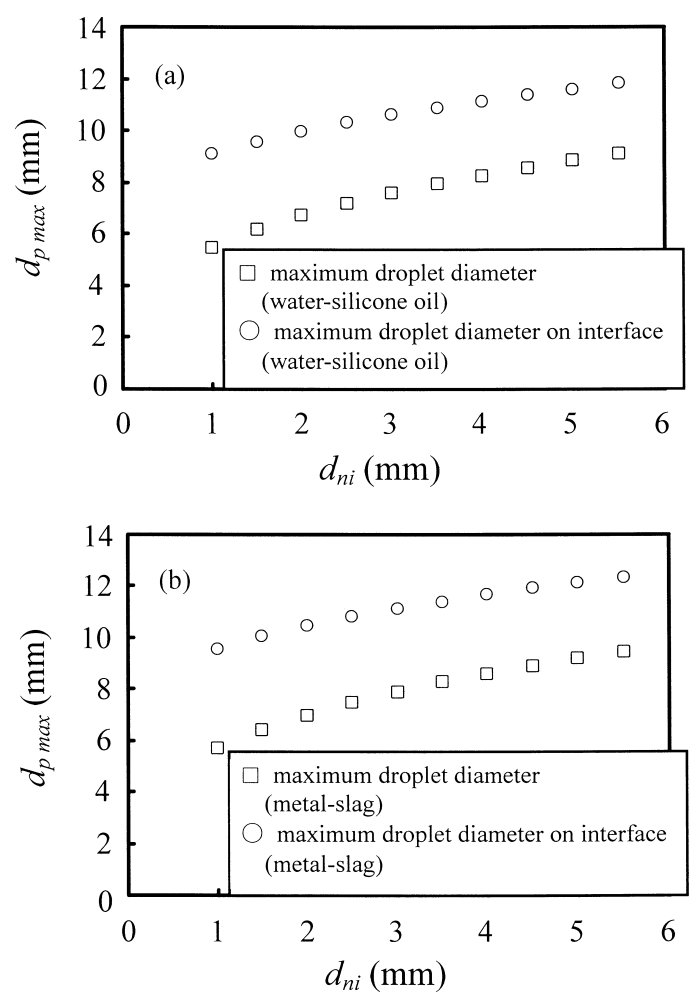

Fig. 8. Maximum droplet diameter growing on liquid-liquid interface. (a) Silicone oil and water system, (b) molten steel and molten slag system.

etrates into the lower layer when its volume reaches approximately three times as large as the initial value.

The difference between the volume of a droplet just before rupture and its initial volume for $H_{\mathrm{n}}=H_{\max }$ is given by

$$
\begin{aligned}
\Delta V & =\pi W_{\max }^{\prime 2} H_{\max } / 6-\pi\left[1.62 d_{\mathrm{ni}} \mathrm{Bo}^{-0.35}\right]^{3 / 6} \\
& =(\pi / 6)\left[W_{\max }^{\prime 2} H_{\max }-4.25 d_{\mathrm{ni}}^{3} \mathrm{Bo}^{-1.05}\right] \ldots .
\end{aligned}
$$

Accordingly, the life of the droplet, $T_{\text {life }}$, is expressed by

$$
\begin{aligned}
T_{\text {life }} & =\Delta V / Q_{\mathrm{w}} \\
& =\left[\pi /\left(6 Q_{\mathrm{w}}\right)\right]\left[W_{\max }^{\prime 2} H_{\mathrm{max}}-4.25 d_{\mathrm{ni}}^{3} \mathrm{Bo}^{-1.05}\right]
\end{aligned}
$$

where $W_{\max }^{\prime}, H_{\max }$ and Bo can be evaluated from Eqs. (6), (8), and (1), respectively.

A very close tendency was obtained for the molten slag and molten steel system, as can be seen in Fig. 8(b). This is because the ratio of $\Delta \rho / \gamma$ in the Bond number and the mod- 


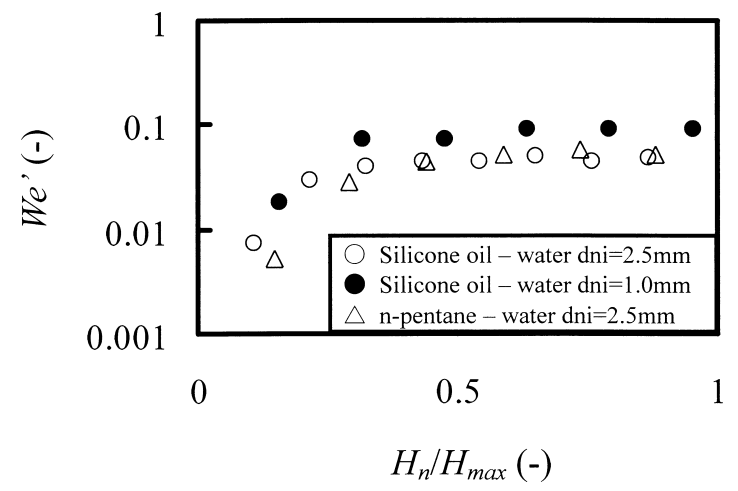

Fig. 9. Relationship between $\mathrm{We}^{\prime}$ and $H_{\mathrm{n}} / H_{\max }$.

ified Weber number (see Eqs. (1) and (2)) has very close values for the two systems. Say, $3190 \mathrm{~s}^{2} / \mathrm{m}^{3}$ for the silicone oil and water system and $2860 \mathrm{~s}^{2} / \mathrm{m}^{3}$ for the real system $\left(\Delta \rho=4000 \mathrm{~kg} / \mathrm{m}^{3}, \gamma=1400 \mathrm{mN} / \mathrm{m}\right)$. The same conclusion can be derived for other Reynolds numbers because the kinematic viscosity of water in Eq. (5) is nearly equal to that of molten steel.

\subsection{Critical Water Flow Rate for the Rupture of Water Droplet on the Interface}

When the water flow rate, $Q_{\mathrm{w}}$, exceeded a certain critical value, a water droplet ruptured as soon as it arrived at the interface and then penetrated into the lower water layer without growing on the interface (see Figs. 4 and 5). As a result, a water column was formed beneath the nozzle. The critical water flow rate for the formation of the water column was non-dimensionalized in terms of the modified Weber number, $\mathrm{We}^{\prime}$, and plotted against the dimensionless distance, $H_{\mathrm{n}} / H_{\max }$, in Fig. 9. The modified Weber number increased with $H_{\mathrm{n}} / H_{\max }$ and approached a constant value of approximately 0.07 . When the distance, $H_{\mathrm{n}} / H_{\max }$, was small, a water column was formed at a very small water flow rate because the interface became unstable as described in the preceding section. The asymptotic critical value of 0.07 was slightly greater than the critical value for the flow in the droplet to become unstable $\left(\mathrm{We}^{\prime}=0.042\right)$ as seen in Fig. 1 . This fact suggests that the formation of a water column is closely associated with the unstable motion in a water droplet.

\subsection{Flows in and around a Droplet after Its Rupture}

It is of essential importance for steel refining to check whether the molten slag in the upper layer is entrapped into the lower molten steel layer when a molten steel droplet ruptures at the slag-metal interface. Figure 10 shows the velocity vectors obtained with the particle image velocimetry. The upper liquid was not entrapped into the lower water layer in relation to the rupture of a water droplet under the present experimental conditions.

The flow patterns associated with the rupture of a water droplet growing on the interface can be classified into three types as schematically shown in Fig. 11. In Type 1 the water issuing out of the nozzle penetrated into the lower water layer without forming vortexes. In Type 2 one vortex was formed in the lower water layer and in Type 3 two vortexes were formed.

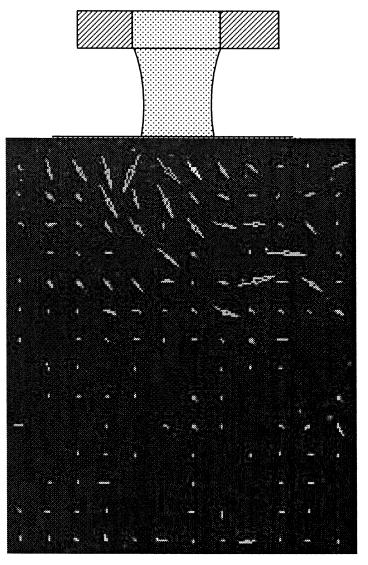

Type 1

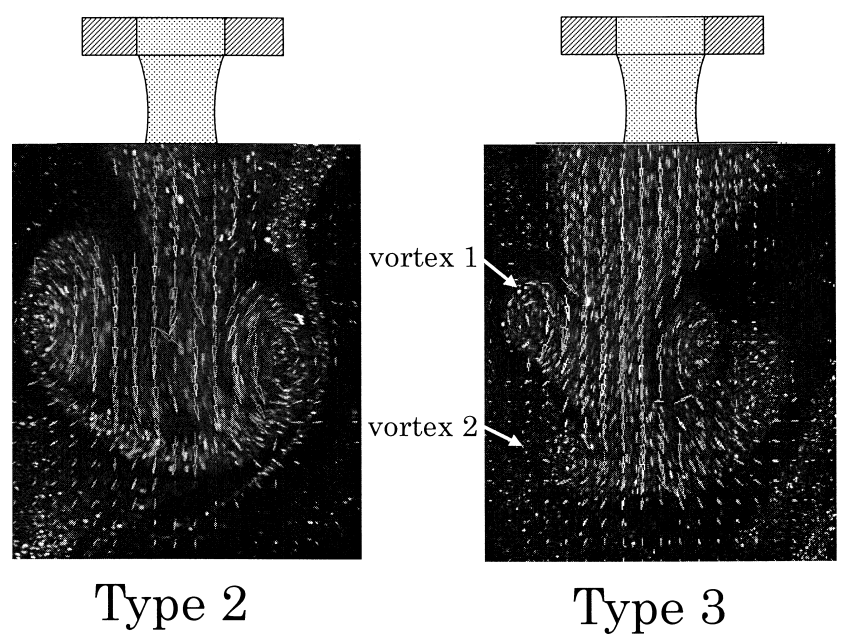

Fig. 10. Flow pattern associated with the rupture of a droplet.

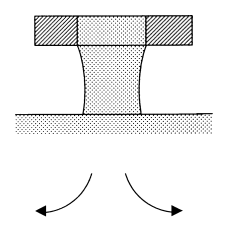

Type 1

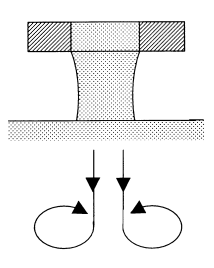

Type 2

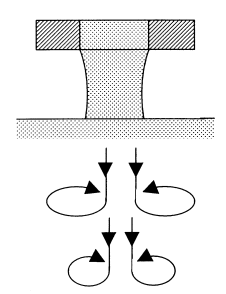

Type 3
Fig. 11. Three types of flow patterns.

Figures 12 and 13 schematically show the flow pattern map as functions of the modified Weber number, $\mathrm{We}^{\prime}$, and the dimensionless distance, $H_{\mathrm{n}} / H_{\mathrm{max}}$, for the water and silicone oil system and water and n-pentane system, respectively. The inner diameter, $d_{\mathrm{ni}}$, was $2.5 \mathrm{~mm}$. Precise velocity measurements were difficult for $d_{\text {ni }}=1.0 \mathrm{~mm}$ because the diameter of a water droplet was too small. Type 1 appeared in the same region for the two systems but Type 3 was not ob- 


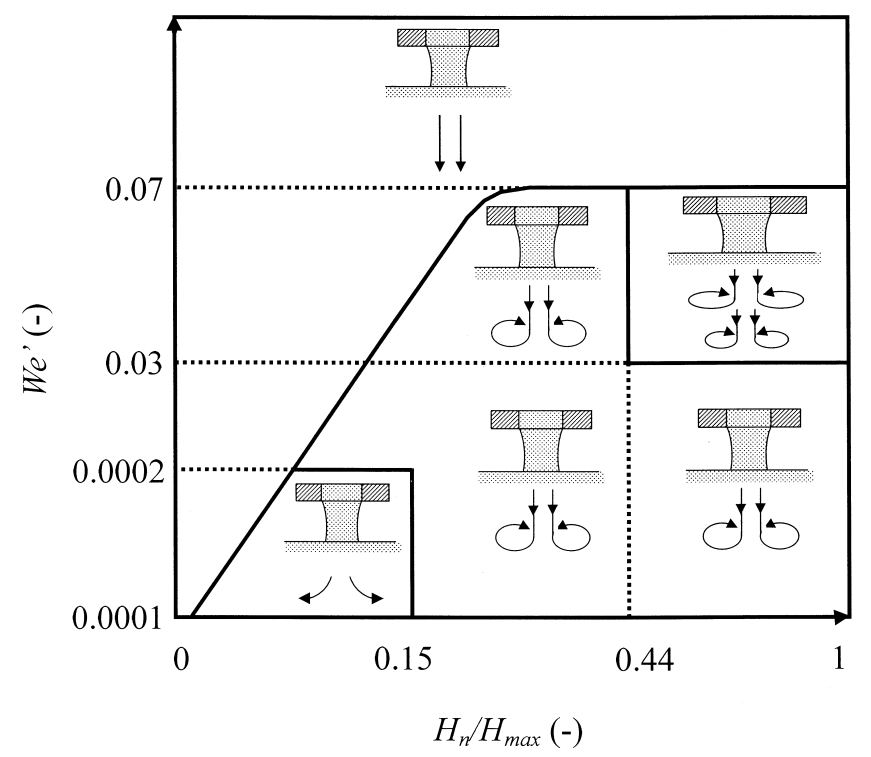

Fig. 12. Flow pattern map for silicone oil and water system.

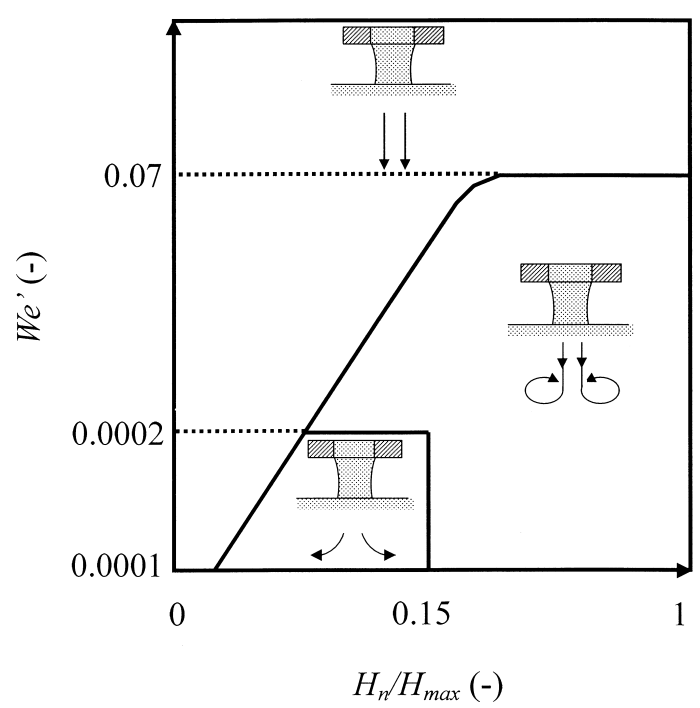

Fig. 13. Flow pattern map for n-pentane and water system.

served for the water and n-pentane system. In order to make clear the reason for the disappearance of Type 3, photographs of a water droplet on the interface are shown for the two systems in Fig. 14. The modified Weber number, $\mathrm{We}^{\prime}$, and the dimensionless distance, $H_{\mathrm{n}} / H_{\mathrm{max}}$, were 0.032 and 0.712 for the silicone oil and water system and 0.033 and 0.886 for the n-pentane and water system, respectively. The size of the droplet is much greater for the water and silicone oil system than for the water and n-pentane system. This result suggests that enough water is not supplied to form two vortexes for the water n-pentane system.

\section{Conclusions}

(1) Empirical Eqs. (7) and (8) were proposed for the maximum width and height of a droplet generated from a single-hole nozzle placed far above the interface, respectively.

(2) An empirical Eq. (9) was derived for the maximum volume of a water droplet growing on a liquid-liquid interface.

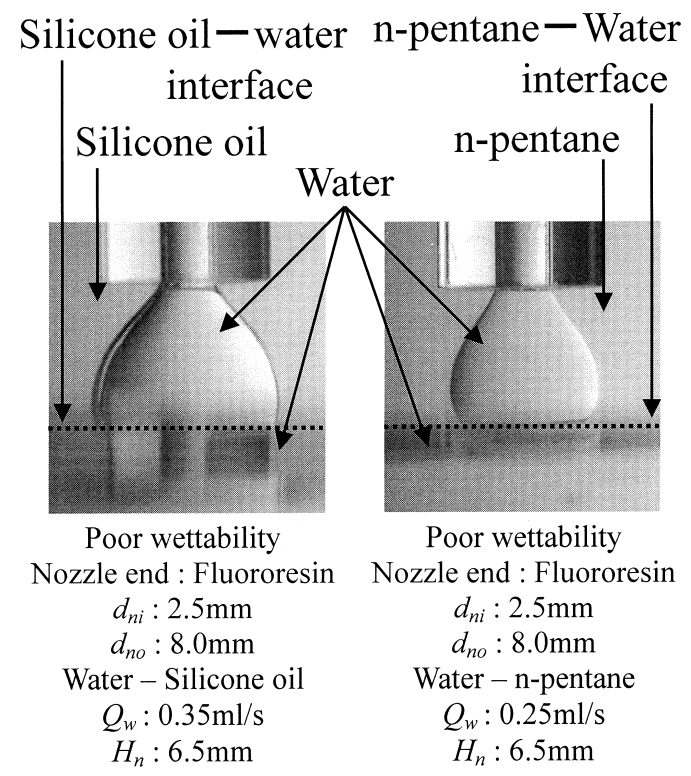

Fig. 14. Droplets growing on liquid-liquid interface.

(3) A water droplet growing on a water and silicone oil interface spread in the horizontal direction due to a silicone oil film formed beneath the droplet. The same phenomenon was observed for the water and n-pentane system.

(4) A water droplet did not grow on the interface for $\mathrm{We}^{\prime}>0.07$ and a water column was formed beneath the nozzle after the rupture of the droplet. The water flow in the droplet was unstable under this condition, as inferred from Fig. 1.

(5) Three types of flow patterns (Types 1, 2, and 3) were observed in the lower water layer in relation to the rupture of a water droplet growing on the interface. No vortex was formed in Type 1. One vortex and two vortexes were generated in Type 2 and Type 3, respectively, as can be seen in Figs. 12 and 13. The upper liquid was not entrapped into the lower liquid layer under the present experimental conditions.

\section{Nomenclature}

Bo: Bond number

$d_{\text {ni }}, d_{\text {no }}$ : Inner and outer diameters of nozzle, respectively

$g$ : Acceleration due to gravity

$H_{\mathrm{n}}$ : Distance between nozzle and interface

$H_{\max }, W_{\max }$ : Maximum height and width of water droplet, respectively

Re : Reynolds number

$Q_{\text {w }}$ : Water flow rate in nozzle

$T_{\text {life }}:$ Life of droplet

$v_{\text {n }}$ : Mean water velocity at nozzle exit

$\mathrm{We}^{\prime}$ : Modified Weber number

$\Delta \rho:$ Density difference

$\gamma:$ Interfacial tension

$\rho_{\mathrm{g}}, \rho_{\mathrm{L}}:$ Gas and liquid densities, respectively

\section{REFERENCES}

1) G. Reiter and K. Schwerdtfeger: ISIJ Int., 32 (1992), No. 1, 50.

2) H. M. Princen: J. Colloid Sci., 18 (1963), 178.

3) H. M. Princen and S. G. Mason: J. Colloid Sci., 29 (1965), 156.

4) S. Hartland: Trans. Inst. Chem. Eng., 45 (1967), 97. 
ISIJ International, Vol. 45 (2005), No. 8

5) S. Hartland, S. Ramakrishnan and R. W. Hartley: Chem. Eng. Sci., 30 (1975), 1141.

6) G. Marrucci: Chem. Eng. Sci., 24 (1969), 975

7) H. Yang, C. C. Park, Y. T. Hu and L. G. Leal: Phys. Fluids, 13 (2001), No. 5, 1087.

8) D. S. Chapman and P. R. Critchlow: J. Fluid Mech., 29 (1967), 177.

9) H. N. Oguz and A. Prosperetti: J. Fluid Mech., 219 (1990), 143.
10) M. Manga and H. A. Stone: J. Fluid Mech., 287 (1995), 279

11) Z. Mohamed-Kassim and E. K. Longmire: Phys. Fluids, 15 (2003), No. 11, 3263.

12) Z. Mohamed-Kassim and E. K. Longmire: Phys. Fluids, 16 (2004), No. 7, 2170.

13) T. Kagawa and M. Iguchi: Mater. Trans., 45 (2004), No. 8, 2703. 